\title{
Dimension Spectrum for Sofic Systems
}

\author{
Jung-Chao Ban, ${ }^{1}$ Chih-Hung Chang, ${ }^{2}$ Ting-Ju Chen, ${ }^{1}$ and Mei-Shao Lin ${ }^{3}$ \\ ${ }^{1}$ Department of Applied Mathematics, National Dong Hwa University, Hualien 970003, Taiwan \\ ${ }^{2}$ Department of Applied Mathematics, Feng Chia University, Taichung 40724, Taiwan \\ ${ }^{3}$ Department of Mathematics, National Central University, Chungli 32054, Taiwan
}

Correspondence should be addressed to Chih-Hung Chang; chihhung@mail.fcu.edu.tw

Received 21 August 2013; Accepted 3 February 2014; Published 10 March 2014

Academic Editor: Christian Maes

Copyright (C) 2014 Jung-Chao Ban et al. This is an open access article distributed under the Creative Commons Attribution License, which permits unrestricted use, distribution, and reproduction in any medium, provided the original work is properly cited.

\begin{abstract}
We study the dimension spectrum of sofic system with the potential functions being matrix valued. For finite-coordinate dependent positive matrix potential, we set up the entropy spectrum by constructing the quasi-Bernoulli measure and the cut-off method is applied to deal with the infinite-coordinate dependent case. We extend this method to nonnegative matrix and give a series of examples of the sofic-affine set on which we can compute the spectrum concretely.
\end{abstract}

\section{Introduction}

Let $\left(\Sigma_{A}, T\right)$ be a subshift of finite type (SFT) with $A$ being the incidence matrix and $T$ being its shift map. Motivated by the study of the iterated function systems (IFS) and generalized Sierpiński carpets (GSC, cf. [1-5]), one considers a special type of potential functions $M: \Sigma_{A} \rightarrow L\left(\mathbb{R}^{d}, \mathbb{R}^{d}\right)$ which take values on the set of $d \times d$ matrices. For $q \in \mathbb{R}$, define the topological pressure as follows:

$$
P_{M}(q)=\lim _{n \rightarrow \infty} \frac{1}{n} \log \sum_{I \in \Sigma_{A, n}} \sup _{x \in[I]}\left\|\prod_{l=0}^{n-1} M\left(T^{l}(x)\right)\right\|^{q},
$$

whenever the limit exists and $\Sigma_{A, n}$ denotes the collection of $n$ cylinders in $\Sigma_{A}$. (Here $\|A\|$ is the matrix norm; that is, $\|A\|=$ $\mathbf{1}_{1 \times d} A \mathbf{1}_{d \times 1}$, where $\mathbf{1}_{d \times 1}$ is the $d \times 1$ column vector with entries being 1's). In [4], if $M$ is positive, that is, $M_{i, j}>0$, and Hölder potential with $\Sigma_{A}$ is topologically mixing, the authors prove that the Gibbs measure for $M$ exists uniquely and the system admits the multifractal analysis. More precisely, let

$$
E_{M}(\alpha)=\left\{x \in \Sigma_{A}: \lim _{n \rightarrow \infty} \frac{\log \left\|\prod_{i=0}^{n-1} M\left(T^{i}(x)\right)\right\|}{n}=\alpha\right\}
$$

be the level set for the upper Lyapunov exponent. Then the Hausdorff dimension of the level set is obtained as follows.
Theorem 1 (see [4, Theorem 1.3]). Let $\left(\Sigma_{A}, T\right)$ be a SFT and let $M: \Sigma_{A} \rightarrow L\left(\mathbb{R}^{d}, \mathbb{R}^{d}\right)$ be Hölder continuous. Then $P_{M}(q)$ is differentiable and for any $\alpha=P_{M}^{\prime}(q), q \neq 0$,

$$
\operatorname{dim}_{H} E_{M}(\alpha)=\frac{1}{\log m}\left\{-\alpha q+P_{M}(q)\right\},
$$

where $\operatorname{dim}_{H}$ denotes the Hausdorff dimension.

The study of the thermodynamic properties with these potentials relates deeply to the fractal properties of the given IFS or GSC. We emphasize that the formula (3) set up the fine structure in the Hausdorff dimension point of view for $\left(\Sigma_{A}, T\right)$. The authors extend this result to the case that $M$ is nonnegative with some additional irreducible conditions' the reader may refer to [3] for the detail. When the underlying space $\mathbf{S}$ is a sofic shift and $d=1$, that is, the potential function is finitary real valued, there raises a natural equilibrium measure called semigroup measure proposed by Kitchens and Tuncel [6]. When $d \geq 2$, the thermodynamic properties relate to fractal dynamics of given sofic affine-invariant sets (cf. [7]).

Theorem 1 investigates the dimension spectrum of SFTs; it is natural to ask whether the formula is preserved by passing to their factors (sofic shifts). To be advanced, does the formula hold for those shifts beyond sofic shifts such as the case for the specification property? Recent research revealed that some properties of SFTs are preserved for the cases beyond the specification (cf. [8-10]). This study intends to show that the 
formula of dimension spectrum of SFTs is passing to their factors, that is, sofic shifts, and their Hausdorff dimension can be expressed explicitly for some class of sofic shifts.

Let $\mathbf{S}$ be a sofic shift which is a subshift of the shift space and let $S$ be the shift map on it. The well-known CurtisLyndon-Hedlund Theorem ([11, Theorem 6.2.9]) demonstrates that if $\phi: \Sigma_{A} \rightarrow S$ is a function, then $\phi$ is a homomorphism if and only if $\phi$ is a sliding block code. The sliding block code is induced from block map, that is, a map $\Phi^{(L)}: \Sigma_{A, L} \rightarrow \mathscr{A}(\mathbf{S})$ for some $L \geq 1$. For all $k \geq 1$ and if $I \in \Sigma_{A, k+L-1}$, define $\Phi_{k}: \Sigma_{A, k+L-1} \rightarrow \mathbf{S}_{k}$ by $\Phi_{k}([I]):=$ $\Phi^{(L)}\left(\pi^{(L)}\left(T^{k-1}([I])\right)\right)$, where $\pi^{(L)}:=\pi_{\mathbb{Z}^{L}}$ is the restriction map of a cylinder to $\mathbb{Z}^{L}$ lattice. Then $\phi: \Sigma_{A} \rightarrow S$ is thus defined as the limit of $\Phi_{k}$; that is, $\phi=\lim _{k \rightarrow \infty} \Phi_{k}$ and $\mathbf{S}:=\{\phi(x)$ : $\left.x \in \Sigma_{A}\right\}$. (We refer reader to [11, 12] for more detail). We call $\phi$ right resolving if for all $I_{1}=\left(i_{1 ; l}\right)_{l=0}^{L-2}$ and any $I_{2}=\left(i_{2 ; l}\right)_{l=0}^{L-2}$ and $I_{3}=\left(i_{3 ; l}\right)_{l=0}^{L-2} \in \Sigma_{A, L-1}$ such that $I_{1} \oplus I_{2}$ and $I_{1} \oplus I_{3} \in \Sigma_{A, L}$ we have

$$
\Phi^{(L)}\left(\left[I_{1} \oplus I_{2}\right]\right) \neq \Phi^{(L)}\left(\left[I_{1} \oplus I_{3}\right]\right),
$$

where $I_{1} \oplus I_{2}=i_{1 ; 0}, i_{1 ; 1}, \ldots, i_{2 ; L-2} \in \Sigma_{A, L}$ if and only if $i_{1 ; l+1}=$ $i_{2 ; l} \forall l=0, \ldots, L-2$. If $I_{1}, I_{2} \in \mathscr{A}\left(\Sigma_{A}\right)$, then we define $I_{1} \oplus I_{2}:=$ $I_{1} I_{2}$. Throughout this paper we assume $\phi: \Sigma_{A} \rightarrow \mathbf{S}$ is right resolving.

In this paper, we study that the dimension spectrum with $N: \mathbf{S} \rightarrow L\left(\mathbb{R}^{d}, \mathbb{R}^{d}\right)$ is a matrix-valued potential on $\mathbf{S}$ taking values on the set of $d \times d$ matrices. To be precise, let $\Gamma_{(+)}^{\star}(\mathbf{S})$ be the collection of $d \times d$ nonnegative (positive) matrices which are $\star$ continuous on $\mathbf{S}$; notation $\star$ stands for the $H$ of Hölder continuous and $C$ for continuous, the same for $\Gamma_{(+)}^{\star}\left(\Sigma_{A}\right)$. For $q \in \mathbb{R}$, let $P_{N}(q)$ be defined similarly as in (1) and the level set for the upper Lyapunov exponent for $N$ is also defined similarly as (2):

$$
E_{N}(\alpha)=\left\{y \in \mathbf{S}: \lim _{n \rightarrow \infty} \frac{\log \left\|\prod_{l=0}^{n-1} N\left(S^{l}([y])\right)\right\|}{n}=\alpha\right\} .
$$

The main results of the present paper are the following. We want to mention here that our results were independently investigated by Feng and Huang [13, Theorem 1.4] via different approach. Our method, except for providing another point of view for the mathematical demonstration, can be applied for evaluating the topological pressure rigorously.

Thearom A. Let $\mathbf{S}$ be a sofic shift induced by $\Sigma_{A}$ and let $N \in$ $\Gamma_{+}^{C}(\mathbf{S})$ be a matrix-valued potential on $\mathbf{S}$ which depends on $k$ coordinates. Then

(1) for all $q \in \mathbb{R} \backslash\{0\}, P_{N}(q)$ is differentiable;

(2) if $\alpha=P_{N}^{\prime}(q)$,

$$
\operatorname{dim}_{H} E_{N}(\alpha)=\frac{1}{\log \lambda}\left\{-\alpha q+P_{N}(q)\right\}
$$

where $\lambda$ is the maximal eigenvalue of $A$.

Theorem A deals with the finite-coordinate dependent matrix potentials. This method also allows us to set up the dimension spectrum for infinite-coordinate dependent one for $\mathbf{S}$. We emphasize here that our method makes the discussion of the limiting measure on infinite-coordinate systems possible. Let

$$
\eta_{n}:=\sup \left\{\frac{M_{i, j}(x)}{M_{i, j}(y)}: I \in \Sigma_{A, n}, x, y \in[I], 1 \leq i, j \leq d\right\} .
$$

Since $M \in \Gamma_{+}^{H}\left(\Sigma_{A}\right)$, we have $\left|\log \eta_{n}\right| \leq C \lambda^{-\alpha n}$ for some $0<$ $\alpha<1$ ([4, Lemma 2.2]). The following result deals with the dimension spectrum for infinite-coordinate $N$.

Thearom B. Let $N \in \Gamma_{+}^{H}(\mathbf{S})$ be a matrix-valued potential on $\mathbf{S}$ which depends on infinite many coordinates. Then

(1) for all $q \in \mathbb{R} \backslash\{0\}, P_{N}(q)$ is differentiable;

(2) if $\alpha=P_{N}^{\prime}(q)$,

$$
\operatorname{dim}_{H} E_{N}(\alpha)=\frac{1}{\log \lambda}\left\{-\alpha q+P_{N}(q)\right\}
$$

where $\lambda$ is the maximal eigenvalue of $A$.

The block map $\Phi^{(L)}$ plays an important role in this method and one of the advantages of this method is that we can prove that (3) holds for $N \in \Gamma_{+}^{H}(\mathbf{S})$ by using the matrix theory argument (Perron-Frobenius Theorem [6]). We will show there are some interesting examples of sofic affine set that we can compute their rigorous formulae for $L^{q}$-spectrum and the pressure functions; then the dimension spectrum is thus derived by simple computation.

The content of the paper is following. In Section 2, we present the proof of Theorem A and the proof of Theorem B is given in Section 3. Section 4 extends Theorems $\mathrm{A}$ and $\mathrm{B}$ to nonnegative matrix-valued potential functions and investigates some examples.

\section{Proof of Theorem A}

This section gives a proof for Theorem A. We recall some definitions first. Denote by $\mathscr{M}\left(\Sigma_{A}\right)$ the set of probability measures on $\Sigma_{A}$ and $\mathscr{M}_{T}\left(\Sigma_{A}\right)$ the subset of $T$-invariant measures of $\mathscr{M}\left(\Sigma_{A}\right), \mathscr{M}(\mathbf{S})$ and $\mathscr{M}_{S}(\mathbf{S})$ are defined similarly.

Definition 2. (1) We say that $\mu \in \mathscr{M}_{T}\left(\Sigma_{A}\right)$ is quasi-Bernoulli if there exists a constant $C>1$ such that

$$
\begin{aligned}
C^{-1} & \mu\left(\left[I_{1}\right]\right) \mu\left(\left[I_{2}\right]\right) \\
& \leq \mu\left(\left[I_{1} I_{2}\right]\right) \\
& \leq C \mu\left(\left[I_{1}\right]\right) \mu\left(\left[I_{2}\right]\right) \quad \forall I_{1}, I_{2} \in \bigcup_{n \in \mathbb{N}} \Sigma_{A, n} .
\end{aligned}
$$

(2) For $q \in \mathbb{R}$, the $L^{q}$-spectrum of $\mu$ is defined by

$$
\tau_{\mu}(q)=\frac{1}{\log \lambda} \liminf _{n \rightarrow \infty} \frac{1}{n} \log \sum_{I \in \Sigma_{A, n}} \mu([I])^{q},
$$

where $\lambda$ denotes the maximal eigenvalue of $A$. 
Our method is motivated by the idea which is proposed in [5] and the intrinsic property of the sliding block codes $\Phi^{(L)}$ and $\phi$; we formulate it briefly.

(1) Since $N$ depends on $k$ coordinates, we construct $\Phi_{k}$ from $\Phi^{(L)}$ as mentioned above. Then the pullback potential on $\Sigma_{A, k+L-1}$ from $\Phi_{k}$ is also defined. We extend the idea of the proof of Lemma 4.3 of [5] to construct an invariant, ergodic probability measure on $\Sigma_{A, k+L-1}$ and extend this measure to some limiting measure which supports the whole $\Sigma_{A}$.

(2) For all $J \in \mathbf{S}_{n}$ we define a measure on $J$ by measuring one of its preimages with the measure in $\Sigma_{A}$ which is constructed in Step 1. Although the measure in $\Sigma_{A}$ satisfies the Markov property and probability properties, the measure on $\mathbf{S}$ cannot share the same properties. However, the space $\mathscr{M}(\mathbf{S})$ is still compact and the standard argument allows us to find an invariant and ergodic measure on $\mathbf{S}$.

(3) Combining steps 1 with 2 we are able to show that the limiting measure is Gibbs-like and satisfies the quasi-Bernoulli property (we emphasize here that this measure is not necessary a Gibbs measure) and the $L^{q}$-spectrum preserved under the factor $\phi$ which is induced from the limit of $\Phi_{k}$; that is, $\phi=\lim _{k \rightarrow \infty} \Phi_{k}$. Therefore, the differentiability and the dimension spectrum can be preserved from $\phi$.

Proof of Theorem A. We divide the proof in the following 4 steps.

Step 1. Let $\Phi^{(L)}: \Sigma_{A, L} \rightarrow \mathscr{A}(\mathbf{S})$ be a sliding block code from $\Sigma_{A, L}$ to $\mathscr{A}(\mathbf{S})$. For $k \geq 1$, define $\Phi_{k}=\Phi^{(k+L-1)}$ from $\Sigma_{A, k+L-1}$ to $\mathbf{S}_{k}$ by $\Phi_{k}([I])=[J]=\left[j_{0}, \ldots, j_{k-1}\right]$ :

$$
j_{l}=\Phi^{(L)}\left(\pi_{[0, L-1]}\left(T^{l-1}([I])\right)\right) \quad \forall l=1, \ldots, k,
$$

where $\pi_{[0, L-1]}([I])=i_{0} i_{1} \cdots i_{L-1}$ denotes the projection map to coordinate $[0, L-1]$ on $\mathbb{Z}^{1}$ for all $I \in \Sigma_{A}$. Define a matrix potential $M$ on $\Sigma_{A, k+L-1}$ by, if $I \in \Sigma_{A, k+L-1}$,

$$
M([I])=N\left(\Phi_{k}([I])\right) .
$$

Then $M$ is well defined for all $I \in \Sigma_{A, k+L-1}$ from the fact that $N$ depends on $k$-coordinate. Write $\Sigma^{(k)}=\Sigma_{A, k+L-1}$. Define $q^{(k)}=\# \Sigma_{A, k+L-1}$ for $k \geq 1$ and $q^{(0)}=\# \mathscr{A}\left(\Sigma_{A}\right)$; we setup a matrix $H^{(k)} \in \mathbf{M}_{d q^{(k-1)} \times d q^{(k-1)}}$ which is indexed by the elements of $\Sigma^{(k-1)}$ as follows: $\forall I_{1}, I_{2} \in \Sigma^{(k-1)}$

$$
H_{I_{1}, I_{2}}^{(k)}= \begin{cases}M\left(\left[I_{1} \oplus I_{2}\right]\right) & \text { if } I_{1} \oplus I_{2} \in \Sigma^{(k)} \\ 0_{d \times d} & \text { otherwise, }\end{cases}
$$

where $0_{d \times d}$ denotes the $d \times d$ matrix with entries which are all zeros. For all $A=\left(A_{i_{1}, i_{2}}\right)_{i_{1}, i_{2}=1}^{n} \in \mathbf{M}_{d n \times d n}(\mathbb{R})$ with $A_{i_{1}, i} \in \mathbf{M}_{d \times d}(\mathbb{R})$, we denote by $I(A)$ the indicator matrix of $A$; that is, $I(A) \in M_{n \times n}(\mathbb{R})$,

$$
I(A)_{i_{1}, i_{2}}= \begin{cases}1 & \text { if } A_{i_{1}, i_{2}} \neq 0_{d \times d} \\ 0 & \text { otherwise. }\end{cases}
$$

It is obvious that if $\Sigma_{A}$ is mixing, then $I(A)$ is primitive. Therefore, if we assume $H=H^{(k)}$, there exists a uniform constant $m>0$ such that for all $I_{1}$ and $I_{2} \in \Sigma^{(k-1)}$ there exists a path $\left(I_{l}^{\prime}\right)_{l=1}^{R}$ with $R \leq m, I_{l}^{\prime} \in \Sigma^{(k-1)}, I_{l}^{\prime}=I_{1}, I_{R}^{\prime}=I_{2}$, and

$$
I^{\prime}:=I_{1}^{\prime} \oplus \cdots \oplus I_{R}^{\prime} \in \Sigma^{(k+R-2)} .
$$

Combining the fact of $N \in \Gamma_{+}^{C}(\mathbf{S})$,

$$
\begin{aligned}
\prod_{l=0}^{R-1} M\left(\pi_{[0, k+L-2]}\left(T^{l}\left(\left[I^{\prime}\right]\right)\right)\right) \\
\quad=\prod_{l=0}^{R-1} N\left(\Phi_{k} \circ \pi_{[0, k+L-2]}\left(T^{l}\left(\left[I^{\prime}\right]\right)\right)\right)>0 .
\end{aligned}
$$

Thus

$$
H_{I_{1}, I_{2}}^{R} \geq \prod_{l=1}^{R-1} M\left(\pi_{[0, k+L-2]}\left(T^{l}\left(\left[I^{\prime}\right]\right)\right)\right)>0,
$$

and also $H^{m}>0$. Let $A^{(k)}=\left\{I^{(l)} \in \Sigma^{(k-1)}: l=\right.$ $\left.1, \ldots, q^{(k-1)}\right\}$ be an ordered set by the lexigraphic ordering and we rearrange $H$ according to this ordering. Since $H$ is primitive, Perron-Frobenius Theorem is applied to show that there exist eigenvalues $\rho_{L}$ and $\rho_{R}>0$ with corresponding eigenvectors $L$ and $R>0$, respectively, for $H$. We may also assume

$$
L=\left(L\left(\left[I^{(l)}\right]\right)\right)_{l=1}^{q^{(k-1)}}, \quad R=\left(R\left(\left[I^{(l)}\right]\right)\right)_{l=1}^{q^{(k-1)}} .
$$

That is,

$$
\begin{aligned}
& (L H)\left(\left[I^{(l)}\right]\right) \\
& =\sum_{\substack{I^{(j)} \in \Sigma^{(k-1):} \\
I^{(j)} \oplus I^{(l)} \in \Sigma^{(k)}}} L\left(\left[I^{(j)}\right]\right) M\left(\left[I^{(j)} \oplus I^{(l)}\right]\right) \\
& =\rho_{L} L\left(\left[I^{(l)}\right]\right) \\
& (H R)\left(\left[I^{(l)}\right]\right) \\
& =\sum_{\substack{I^{(j)} \in \Sigma^{(k-1):} \\
I^{(l)} \oplus I^{(j)} \in \Sigma^{(k)}}} M\left(\left[I^{(l)} \oplus I^{(j)}\right]\right) R\left(\left[I^{(j)}\right]\right) \\
& =\rho_{R} R\left(\left[I^{(l)}\right]\right) .
\end{aligned}
$$

For all $I \in \Sigma^{(k+j)}$ and $j \geq 0$, let $\pi^{(k)}=\pi_{[0, k+L-2]}$ and

$$
\prod_{[I]} M=\prod_{l=0}^{j-1} M\left(\pi^{(k)}\left(T^{l}([I])\right)\right) .
$$


We define a measure as follows:

$\eta_{L}([I])$

$$
=\rho_{L}^{-j} L\left(\pi^{(k-1)}([I])\right)\left[\prod_{[I]} M\right] R\left(\pi^{(k-1)}\left(T^{j-1}([I])\right)\right),
$$

$\eta_{R}([I])$

$$
=\rho_{R}^{-j} L\left(\pi^{(k-1)}([I])\right)\left[\prod_{[I]} M\right] R\left(\pi^{(k-1)}\left(T^{j-1}([I])\right)\right) .
$$

It follows from (19) that if $I \in \Sigma^{(k+j-1)}$,

$$
\begin{aligned}
& \sum_{\substack{I_{1} \in \Sigma^{(k-1):} \\
I_{1} \oplus I \in \Sigma^{(k+j)}}} \eta_{L}\left(\left[I_{1} \oplus I\right]\right) \\
& =\sum_{\substack{I_{1} \in \Sigma^{(k-1):} \\
I_{1} \oplus I \in \Sigma^{(k+j)}}} \rho_{L}^{-j} L\left(\pi^{(k-1)}\left(\left[I_{1} \oplus I\right]\right)\right)\left[\prod_{\left[I_{1} \oplus I\right]} M\right] \\
& \times R\left(\pi^{(k-1)}\left(T^{j-1}\left(\left[I_{1} \oplus I\right]\right)\right)\right) \\
& =\rho_{L}^{-j} \sum_{\substack{I_{1} \in \Sigma^{(k-1):} \\
I_{1} \oplus I \in \Sigma^{(k+j)}}} L\left(\pi^{(k-1)}\left(\left[I_{1}\right]\right)\right) M\left(\pi^{(k)}\left(\left[I_{1} \oplus I\right]\right)\right) \\
& \times\left[\prod_{[I]} M\right] R\left(\pi^{(k-1)}\left(T^{j-1}\left(\left[I_{1} \oplus I\right]\right)\right)\right) \\
& =\rho_{L}^{-(j-1)} L\left(\pi^{(k-1)}([I])\right)\left[\prod_{[I]} M\right] \\
& \times R\left(\pi^{(k-1)}\left(T^{j-1}\left(\left[I_{1} \oplus I\right]\right)\right)\right) \\
& =\rho_{L}^{-(j-1)} L\left(\pi^{(k-1)}([I])\right)\left[\prod_{[I]} M\right] \\
& \times R\left(\pi^{(k-1)}\left(T^{j-2}([I])\right)\right) \\
& =\eta_{L}([I]) .
\end{aligned}
$$

That is,

$$
\eta_{L}([I])=\sum_{I_{1} \in \Sigma^{(k-1)}: I_{1} \oplus I \in \Sigma^{(k+j)}} \eta_{L}\left(\left[I_{1} \oplus I\right]\right) .
$$

It follows from the same computation we also have that

$$
\eta_{R}([I])=\sum_{I_{1} \in \Sigma^{(k-1)}: I \oplus I_{1} \in \Sigma^{(k+j)}} \eta_{R}\left(\left[I \oplus I_{1}\right]\right) .
$$

This implies that $\forall n \geq 0$,

$$
\begin{gathered}
\sum_{I \in \Sigma^{(k+n)}} \eta_{L}([I])=\sum_{I \in \Sigma^{(k)}} \eta_{L}([I]), \\
\sum_{I \in \Sigma^{(k+n)}} \eta_{R}([I])=\sum_{I \in \Sigma^{(k)}} \eta_{R}([I]) .
\end{gathered}
$$

It can be easily checked that $\rho_{L}=\rho_{R}$ and then we define

$$
\eta([I])=\eta_{L} \eta_{(k)}^{-1}, \quad \text { where } \eta_{(k)}=\sum_{I \in \Sigma^{(k)}} \eta_{L}([I]) .
$$

The Kolmogorov consistence theorem is applied to show that there exists a measure $\mu$ on $\Sigma_{A}$ such that

$$
\mu([I])=\eta([I]) \quad \forall I \in \cup_{n \geq 0} \Sigma^{(k+n)} .
$$

Step 2. In this step, we will define a measure on $\mathscr{M}_{S}(\mathbf{S})$. Since

$$
\Phi_{k+j}\left(\Sigma^{(k+j)}\right)=\mathbf{S}_{k+j}
$$

is onto, for all $J \in \mathbf{S}_{k+j}$, we define an ordered set as

$$
P_{J}:=\left\{I^{(l)} \in \Sigma^{(k+j)}: \Phi_{k+j}\left(\left[I^{(l)}\right]\right)=J\right\}
$$

and set a measure on $\mathbf{S}$ :

$$
\xi_{1}([J])=\eta_{L}\left(\left[I^{(1)}\right]\right), \quad \forall J \in \mathbf{S}_{k+j}, I^{(1)} \in P_{J} .
$$

We note here that $\xi_{1}$ is not invariant. Let

$$
\xi([J])=\xi_{1}([J]) \xi_{(k)}^{-1} \quad \text { where } \xi_{(k)}=\sum_{J \in \mathbf{S}_{k}} \xi_{1}([J])
$$

for all $J \in \mathbf{S}_{k+j}$ and assume $I_{1}, I_{2} \in P_{J}$,

$$
\begin{array}{r}
\Phi_{k}\left(\pi^{(k)}\left(T^{l}\left(\left[I_{1}\right]\right)\right)\right)=\Phi_{k}\left(\pi^{(k)}\left(T^{l}\left(\left[I_{2}\right]\right)\right)\right) \\
\forall l=0, \ldots, j-1 .
\end{array}
$$

Hence

$$
\begin{aligned}
& \prod_{l=0}^{j-1} M\left(\pi^{(k)}\left(T^{l}\left(\left[I_{1}\right]\right)\right)\right) \\
& =\prod_{l=0}^{j-1} N\left(\Phi_{k}\left(\pi^{(k)}\left(T^{l}\left(\left[I_{1}\right]\right)\right)\right)\right) \\
& =\prod_{l=0}^{j-1} N\left(\Phi_{k}\left(\pi^{(k)}\left(T^{l}\left(\left[I_{2}\right]\right)\right)\right)\right) \\
& =\prod_{l=0}^{j-1} M\left(\pi^{(k)}\left(T^{l}\left(\left[I_{2}\right]\right)\right)\right) .
\end{aligned}
$$

Set

$$
\begin{aligned}
U_{L} & =\max _{i=1, \ldots, d_{I}} \max _{I \in \Sigma^{(k-1)}} L_{i}([I]), \\
U_{R} & =\max _{i=1, \ldots, d_{I}} \max _{I \in \Sigma^{(k-1)}} R_{i}([I]), \\
V_{L} & =\min _{i=1, \ldots, d_{I}} \min _{I \in \Sigma^{(k-1)}} L_{i}([I]), \\
V_{R} & =\min _{i=1, \ldots, d_{I \in \Sigma^{(k-1)}}} R_{i}([I]),
\end{aligned}
$$

where $B_{i}([I])$ denotes the $i$-coordinate of vector of $B([I])$ for $B=L$ or $R$. Since $\phi=\lim _{k \rightarrow \infty} \Phi_{k}$ is right resolving, for all 
$J \in \mathbf{S}_{n}$ there is at least one and at most $K$ preimages of $I \in \Sigma^{(n)}$ such that $\Phi_{n}([I])=J$. Therefore, if $j \geq 0$ and $J \in \mathbf{S}_{k+j}$, it follows from (33) that

$$
\begin{aligned}
& \rho^{-j} V_{L} V_{R}\left\|\prod_{l=0}^{j-1} M\left(\pi^{(k)}\left(T^{l}([I])\right)\right)\right\| \\
& \leq \eta_{L}([I]) \leq \eta_{L}\left(\Phi_{k+j}^{-1}([J])\right) \\
& =\eta_{L}\left(\bigcup_{I \in P_{J}}[I]\right) \leq \sum_{I \in P_{J}} \eta_{L}([I]) \\
& \leq \rho^{-j} K U_{L} U_{R}\left\|\prod_{l=0}^{j-1} M\left(\pi^{(k)}\left(T^{l}([I])\right)\right)\right\| .
\end{aligned}
$$

By the positivity of $L=\left(L\left(\left[I^{(l)}\right]\right)\right)_{l=1}^{q^{(k-1)}}, R=\left(R\left(\left[I^{(l)}\right]\right)\right)_{l=1}^{q^{(k-1)}}$, and $q^{(k-1)}$ is finite, we can conclude that there exist two constants $P$ and $Q>0$ such that $1 \leq U_{L} / V_{L} \leq P$ and $1 \leq U_{R} / V_{R} \leq Q$; then

$$
\begin{aligned}
\eta_{L} & \left(\Phi_{k+j}^{-1}([J])\right) \\
& \leq \rho^{-j} K U_{L} U_{R}\left\|\prod_{l=0}^{j-1} M\left(\pi^{(k)}\left(T^{l}([I])\right)\right)\right\| \\
& \leq \rho^{-j} K P Q V_{L} V_{R}\left\|\prod_{l=0}^{j-1} M\left(\pi^{(k)}\left(T^{l}([I])\right)\right)\right\| \\
& \leq \rho^{-j} K P Q L\left(\pi^{(k-1)}([I])\right) \\
& \times \prod_{l=0}^{j-1} M\left(\pi^{(k)}\left(T^{l}([I])\right)\right) R\left(\pi^{(k-1)}\left(T^{j-1}([I])\right)\right) \\
& =K P Q \xi_{1}([J]), \\
\eta_{L} & \left(\Phi_{k+j}^{-1}([J])\right) \geq \rho^{-j} V_{L} V_{R}\left\|\prod_{l=0}^{j-1} M\left(\pi^{(k)}\left(T^{l}([I])\right)\right)\right\| \\
& \geq P^{-1} Q^{-1} \xi_{1}([J]) .
\end{aligned}
$$

This means that for all $J \in \mathbf{S}_{k}$ there exists a constant $C_{1}>0$ such that

$$
C_{1}^{-1} \xi_{1}([J]) \leq \eta_{L}\left(\Phi_{k}^{-1}([J])\right) \leq C_{1} \xi_{1}([J])
$$

And thus

$$
C_{2}^{-1} \xi([J]) \leq \eta\left(\Phi_{k}^{-1}([J])\right) \leq C_{2} \xi([J])
$$

for some $C_{2}>0$.

Step 3. Since $\xi$ is not invariant, we follow the proof of [4] to construct an invariant and ergodic measure satisfying the property of (21) in this step. For all $J \in \mathbf{S}_{k}$, define a sequence $\left\{\sum_{l=0}^{n-1} \xi \circ S^{-l}([J])\right\}_{n \geq 1}$. It follows from (37) that if $l \geq 0$,

$$
\begin{aligned}
\xi_{1} & \circ S^{-l}([J]) \\
& =\sum_{J_{1}: J_{1} J \in \mathbf{S}_{l+k}} \xi_{1}\left(\left[J_{1} J\right]\right) \\
& =\sum_{I_{1}: I_{1} I \in P_{I_{1} J}} \eta_{L}\left(\left[I_{1} I\right]\right) \\
& \leq \sum_{J_{1}: J_{1} J \in \mathbf{S}_{l+k}} \eta_{L}\left(\Phi_{l+k}^{-1}\left(\left[J_{1} J\right]\right)\right)=\eta_{L}\left(\Phi_{k}^{-1}[J]\right) .
\end{aligned}
$$

Hence there exists a constant $C_{3}>0$ such that

$$
C_{3}^{-1} \eta_{L}\left(\Phi_{k}^{-1}[J]\right) \leq \xi_{1} \circ S^{-l}([J]) \leq C_{3} \eta_{L}\left(\Phi_{k}^{-1}[J]\right)
$$

Thus there exists a $C_{4}>0$ such that

$$
C_{4}^{-1} \eta\left(\Phi_{k}^{-1}([J])\right) \leq \xi \circ S^{-l}([J]) \leq C_{4} \eta\left(\Phi_{k}^{-1}([J])\right)
$$

Since $\mathbf{S}$ is compact, then let $v \in \mathscr{M}(\mathbf{S})$ be the limiting measure of

$$
\left\{\sum_{l=0}^{n-1} \xi \circ S^{-l}([J])\right\}_{n \geq 1} .
$$

Combining the fact that $\lim _{n \rightarrow \infty} \Phi_{k+n}=\phi$ with the above computations it yields $\nu \ll \mu$ and $\mu \ll \nu$. Up to a small modification of the proof in Theorem 1.1 of [4] we also have that $v$ is ergodic. The Radon-Nikodym theorem applies to show that there is a constant $C>0$ such that $\nu([J])=$ $C \mu\left(\Phi_{l}^{-1}([J])\right)$ for $\nu$-a.e. $J \in S_{l}$ and $l \geq k$. It follows from that $\nu$ and $\mu$ are both invariant probability measures. We obtain $C=1$ and for all $J \in \mathbf{S}$

$$
\nu([J])=\lim _{l \rightarrow \infty} \mu\left(\Phi_{l}^{-1}([J])\right)=\mu\left(\phi^{-1}([J])\right) .
$$

Step 4. From the above computation we obtain that if $J \in \mathbf{S}_{l}$ with $l \geq k$ and $I^{(1)} \in P_{J}$, then $\nu([J])=\mu\left(\left[I^{(1)}\right]\right)=\eta\left(\left[I^{(1)}\right]\right)$. Moreover, there exists $Q_{1}>0$ such that

$$
Q_{1}^{-1} \eta_{L}\left(\left[I^{(1)}\right]\right) \leq \eta\left(\left[I^{(1)}\right]\right) \leq Q_{1} \eta_{L}\left(\left[I^{(1)}\right]\right) .
$$

With the positivity of $M$ implements there exists a constant $Q_{2}>0$ such that for any $x \in \Sigma_{A}, n, l \in \mathbb{N}$ we have

$$
\begin{aligned}
Q_{2}^{-1} & \left\|M(x) \cdots M\left(T^{n-1}(x)\right)\right\| \\
& \cdot\left\|M\left(T^{n}(x)\right) \cdots M\left(T^{n+l-1}(x)\right)\right\| \\
\leq & \left\|M(x) \cdots M\left(T^{n+l-1}(x)\right)\right\| \quad(\text { Lemma } 2.1 \text { of }[4]) \\
\leq & Q_{2}\left\|M(x) \cdots M\left(T^{n-1}(x)\right)\right\| \\
& \cdot\left\|M\left(T^{n}(x)\right) \cdots M\left(T^{n+l-1}(x)\right)\right\| .
\end{aligned}
$$


This demonstrates $\eta_{L}$ is quasi-Bernoulli and so are $\eta$ and $\mu$. Hence $v \in \mathscr{M}_{S}(\mathbf{S})$ is a quasi-Bernoulli measure. According to the fact that right-resolving factor $\phi$ cannot increase the topological entropy, we can assert that

$$
\tau_{\nu}(q)=\tau_{\phi_{*} \mu}(q)=\tau_{\mu}(q) \quad \text { for any } q \in \mathbb{R},
$$

where $\phi_{*} \mu=\mu\left(\phi^{-1}\right)$. Theorem 1.3 of [4] is applied to show that for all $q \in \mathbb{R} \backslash\{0\}, \tau_{v}$ is differentiable and if $\alpha=P_{N}^{\prime}(q)$,

$$
\operatorname{dim}_{H} E_{N}(\alpha)=\frac{1}{\log \lambda}\left(-\alpha q+P_{N}(q)\right)
$$

where $\lambda$ denotes the maximal eigenvalue of $A$. Finally, the differentiability for $P_{N}(q)$ with $q \neq 0$ comes from the fact $P_{N}(q)=P_{M}(q)$ since $\phi$ is right resolving and $M$ is the pullback potential of $N$. This completes the proof.

Remark 3. We remark that in the proof of Theorem A, $v \in$ $\mathscr{M}_{S}(\mathbf{S})$ is not a Gibbs measure, and in the following, we will show that this method allow us to approximate the potential depending on infinite coordinate for $N \in \Gamma_{+}^{H}(\mathbf{S})$.

\section{Proof of Theorem B}

In this section, we extend our result to the matrix-valued potentials that are infinite-coordinate dependent.

Proof of Theorem B. The first statement is an immediate consequence of Theorem A since $N^{(k)}$ depends on $k$-coordinate. It is still remaining to prove the second statement.

For $k \geq 1, I \in \Sigma^{(k)}$ with $I \in P_{J}$, let $H^{(k)} \in \mathbf{M}_{d q^{(k-1)} \times d q^{(k-1)}}$ $(\mathbb{R})$ and let $\rho^{(k)}$ be its maximal eigenvalue as in Theorem A. Since $I\left(H^{(k)}\right)$ is primitive and $M^{(k)}$ is positive, $H^{(k)}$ is also primitive for all $k \in \mathbb{N}$. We claim that $\eta_{k}^{-1} \rho^{(k)} \leq \rho^{(k+1)} \leq \eta_{k} \rho^{(k)}$ for $k \geq 1$. Indeed, let $H^{(k)}$ and $H^{(k+1)}$ be indexed by $\Sigma^{(k-1)}$ and $\Sigma^{(k)}$, respectively. For all $I \in \Sigma^{(k+1)},[J]=\left[j_{0} \cdots j_{k}\right]:=$ $\Phi_{k+1}([I])$,

$$
\begin{aligned}
& M^{(k+1)}([I]) \\
& \quad:=N^{(k+1)}\left(\Phi_{k+1}([I])\right)=\max _{y \in\left[j_{0} \cdots j_{k}\right]} N(y) \\
& \quad \leq \eta_{k} \max _{y \in\left[j_{0} j_{1} \cdots j_{k-1}\right]} N(y)=\eta_{k} N^{(k)}\left(\left[J^{*}\right]\right)=\eta_{k} M^{(k)}\left(\left[I^{*}\right]\right),
\end{aligned}
$$

where $J^{*}:=j_{0} \cdots j_{k-1}$ and $I^{*}:=i_{0} \cdots i_{k+L-1}$. Therefore, for $m \geq 1$,

$$
\begin{aligned}
& \left\|H^{(k+1) m}\right\| \\
& =\sum_{I \in \Sigma^{(k+m)}}\left\|\prod_{l=0}^{m-1} M^{(k+1)}\left(\pi^{(k+1)}\left(T^{l}([I])\right)\right)\right\| \\
& \leq\left(\eta_{k}\right)^{m} \sum_{I \in \Sigma^{(k+m)}}\left\|\prod_{l=0}^{m-1} M^{(k)}\left(\pi^{(k)}\left(T^{l}([I])\right)\right)\right\|
\end{aligned}
$$

$$
\begin{aligned}
& \leq C\left(\eta_{k}\right)^{m} \sum_{I \in \Sigma^{(k+m-1)}}\left\|\prod_{l=0}^{m-1} M^{(k)}\left(\pi^{(k)}\left(T^{l}([I])\right)\right)\right\| \\
& =C\left(\eta_{k}\right)^{m}\left\|H^{(k) m}\right\|,
\end{aligned}
$$

for some $C>1$. Hence, $\left\|H^{(k+1) m}\right\|^{1 / m} \leq C^{1 / m}\left(\eta_{k}\right)\left\|H^{(k) m}\right\|^{1 / m}$. Taking $m \rightarrow \infty$ we have

$$
\rho^{(k+1)} \leq \eta_{k} \rho^{(k)}, \quad \text { for } k \geq 1 .
$$

Using the same argument, we also have

$$
\rho^{(k+1)} \geq \eta_{k}^{-1} \rho^{(k)}, \quad \text { for } k \geq 1 \text {. }
$$

On the other hand, for $I \in \Sigma^{(k+j)}$ with $j \geq 0$ being fixed,

$$
\begin{aligned}
\eta_{L}^{(k+1)}([I]) & \left(\rho^{(k+1)}\right)^{-j} L\left(\pi^{(k)}([I])\right) \\
& \times\left[\prod_{[I]} M^{(k+1)}\right] R\left(\pi^{(k)}\left(T^{j-1}([I])\right)\right) \\
\leq & D \times\left(\rho^{(k+1)}\right)^{-j}\left\|\prod_{[I]} M^{(k+1)}\right\| \\
\leq & D \times\left(\rho^{(k)}\right)^{-j} \eta_{k}^{j}\left\|\prod_{[I]} M^{(k+1)}\right\| \quad(\mathrm{By}(51) \| \\
\leq & D^{\prime} \times\left(\rho^{(k)}\right)^{-j} \eta_{k}^{j} \eta_{k}^{j}\left\|\prod_{[I]} M^{(k)}\right\| \\
\leq & D^{\prime \prime} \eta_{k}^{2 j} \eta_{L}^{(k)}([I]),
\end{aligned}
$$

for some $D, D^{\prime}, D^{\prime \prime}>0$. Similarly we have

$$
\eta_{L}^{(k+1)}([I]) \geq\left(D^{\prime \prime} \eta_{k}^{2 j}\right)^{-1} \eta_{L}^{(k)}([I])
$$

and there exists $D_{1}>0$ such that

$$
\left(D_{1} \eta_{k}^{2 j}\right)^{-1} \mu^{(k)}([I]) \leq \mu^{(k+1)}([I]) \leq D_{1} \eta_{k}^{2 j} \mu^{(k)}([I]) .
$$

The fact that $\lim _{k \rightarrow \infty} \eta_{k}=1$ asserts and there exists $D_{2}>$ $0, n \in \mathbb{N}$ such that for $k \geq n$ we have

$$
D_{2}^{-1} \mu^{(k)}([I]) \leq \mu^{(k+1)}([I]) \leq D_{2} \mu^{(k)}([I]) .
$$

This demonstrates $\mu^{(k)} \rightarrow \tilde{\mu}$ as $k \rightarrow \infty$ for some $\tilde{\mu}$. Define $\tilde{\nu}=\phi_{*} \tilde{\mu} \cdot v^{(k)}=\phi_{*} \mu^{(k)}$ implies

$$
\lim _{k \rightarrow \infty} \nu^{(k)}=\lim _{k \rightarrow \infty} \phi_{*} \mu^{(k)}=\phi_{*} \tilde{\mu}=\widetilde{v} .
$$

It can also be checked that $\tilde{\mu}$ satisfies the quasi-Bernoulli property and for all $q \in \mathbb{R} \backslash\{0\}$,

$$
\tau_{\widetilde{\mu}}(q)=\lim _{k \rightarrow \infty} \tau_{\mu^{(k)}}(q) .
$$


Using the same proof of Theorem A, we have

$$
\begin{aligned}
\tau_{\widetilde{\mu}}(q) & =\lim _{k \rightarrow \infty} \tau_{\mu^{(k)}}(q)=\lim _{k \rightarrow \infty} \tau_{\phi_{*} \mu^{(k)}}(q) \\
& =\lim _{k \rightarrow \infty} \tau_{\gamma^{(k)}}(q)=\tau_{\widetilde{v}}(q)
\end{aligned}
$$

Combining Theorems 1 with $\mathrm{A}$, we conclude that $P_{N}(q)$ is thus differentiable and the desired equality (8) follows. This completes the proof.

\section{Examples}

This section illustrates several examples that help for the understanding of our results.

4.1. Computation of Dimension Spectrum. Suppose $X$ is an irreducible subshift of finite type and $\pi: X \rightarrow Y$ is a factor. Chazottes and Ugalde [14] indicate that if a matrix-valued push-forward potential function $\mathbf{N}$ is row allowable and is positive on periodic points, then there exists a unique Gibbs measure on $Y$. Here $\mathbf{N}$ is called row allowable if there is no zero row in $\mathbf{N}$. Before extending our results to nonnegative matrix-valued potential functions, we give the definition of a column allowable matrix first.

Definition 4. We call $A \in \mathbf{M}_{n \times n}(\mathbb{R})$ column allowable if for all $1 \leq j \leq n$, we have $\sum_{i=1}^{n} A_{i j} \geq 1$. We also denote by $\mathcal{N}_{n}$ the collection of column allowable matrices of size $n \times n$.

It can be easily verified that $\mathcal{N}_{n}$ forms a semigroup under matrix product.

Lemma 5. If $A$ and $B \in \mathcal{N}_{n}$, then $A B \in \mathcal{N}_{n}$.

Proof. Indeed, for all $j=1, \ldots, n$,

$$
\begin{aligned}
& \sum_{i=1}^{n}(A B)_{i j} \\
& =\sum_{i=1}^{n} \sum_{k=1}^{n} A_{i k} B_{k j} \\
& =\sum_{k=1}^{n} B_{k j}\left(\sum_{i=1}^{n} A_{i k}\right) \\
& \geq \sum_{k=1}^{n} B_{k j} \geq 1 .
\end{aligned}
$$

This completes the proof.

For nonnegative matrix-valued potential $N$, we have the following result.

Theorem 6. Let $N \in \Gamma^{H}(\mathbf{S}) \cap \mathcal{N}_{d}$ depend on $k$-coordinate and there exists a finite set $\Lambda \subset \bigcup_{n \geq k} \Sigma^{(n)}=: \Sigma^{*}$ such that for all $I_{1}$ and $I_{2} \in \Sigma^{*}$ there exists $I \in \Lambda$ such that $I_{1} \oplus I \oplus I_{2} \in \Sigma^{*}$ and $M([I])=N\left(\Phi_{k}([I])\right)>0$ for all $I \in \Lambda$, and then (6) holds.
Proof. We give the proof for the case that all elements in $\Lambda$ are equal length and the case for different length is in the same fashion. It follows from the proof in Theorem A that $H^{(k)}$ can be constructed which is indexed by the $\Sigma^{(k)}$. Since for any $\omega \epsilon$ $\Lambda,|\omega|=k+L-1$ (we assume that $\Lambda$ is equal length and the definition $\Lambda \subset \bigcup_{n \geq k} \Sigma^{(n)}$ allows us to define all elements which have equal length of $k+L-1$ ) we also assume that $\Lambda$ consist of only one element; say $I^{*}$. Without loss of generality, assume $I^{*} \in \Sigma^{(k-1)}$. It suffices to show that $H^{(k)}$ is primitive. Indeed, for any $I_{1}$ and $I_{2} \in \Sigma^{(k-1)}$, since $N \in \mathcal{N}_{d}$, Lemma 5 is thus applied to show that

$$
\begin{aligned}
H_{I_{1}, I_{2}}^{2} & =M\left(\left[I_{1} \oplus I^{*}\right]\right) M\left(\left[I^{*} \oplus I_{2}\right]\right) \\
& =N\left(\Phi_{k}\left(\left[I_{1} \oplus I^{*}\right]\right)\right) N\left(\Phi_{k}\left(\left[I^{*} \oplus I_{2}\right]\right)\right)>0 .
\end{aligned}
$$

This means that $H^{2}>0$. The other case can be done similarly. Therefore, the same proof as in Theorem A leads to (6) and the proof is completed.

In the proof of Theorem $\mathrm{A}$, the $L^{q}$-spectrum plays an important role for the computing of dimension spectrum. We emphasize that for a measure $\mu \in \mathscr{M}_{T}\left(\Sigma_{A}\right)$, it is not easy to compute the rigorous formula for $\tau_{\mu}$. If the measure $\mu$ is given as in Theorem $\mathrm{A}$, the following theorem provides a class of matrix-valued potentials for which we can compute its $L^{q}$-spectrum explicitly. Let $N \in \Gamma^{H}(\mathbf{S}) \cap \mathcal{N}_{d}$ depend on $k$-coordinate and $H:=H^{(k)}$ as defined in Theorem A; we define a matrix $\mathbf{R}(q) \in \mathbf{M}_{q^{(k-1)} \times q^{(k-1)}}$ from $H$ (recall that $\left.q^{(k)}=\# \Sigma_{A, k+L-1}\right)$ as follows:

$$
\mathbf{R}(q)_{I_{1}, I_{2}}= \begin{cases}\rho\left(M\left(\left[I_{1} \oplus I_{2}\right]\right)\right)^{q}, & \text { if } I_{1} \oplus I_{2} \in \Sigma^{(k)} \\ 0, & \text { otherwise. }\end{cases}
$$

$\rho(A) \in \mathbb{R}$ denotes the maximal eigenvalue of $A \in \mathbf{M}_{d \times d}(\mathbb{R})$.

Proposition 7. Under the same assumptions of Theorem 6, assume that

$$
N=\{N([J])\}_{J \in \mathbf{S}_{k}} \in \Gamma^{H}(\mathbf{S})
$$

satisfies that

$$
N\left(\left[J_{1}\right]\right) N\left(\left[J_{2}\right]\right)=N\left(\left[J_{2}\right]\right) N\left(\left[J_{1}\right]\right) \quad \forall J_{1} \neq J_{2} \in \mathbf{S}_{k} .
$$

Assume that $H^{(k)}$ and $\nu \in \mathscr{M}_{S}(\mathbf{S})$ are as defined in Theorem $A$. Then

$$
\tau_{\nu}(q)=\frac{1}{\log \lambda}(-q \log \rho+\log \Theta(q))
$$

where $\Theta(q)$ is the maximal root of the characteristic polynomial of $\mathbf{R}(q) \in \mathbf{M}_{q^{(k-1)} \times q^{(k-1)}}$.

Proof. Let

$$
H=\left[M\left(\left[I_{1} \oplus I_{2}\right]\right)\right]_{I_{1}, I_{2} \in \Sigma^{(k-1)}}
$$

be constructed as in Step 1 of the proof of Theorem A. Since elements of $N$ are mutually commuted, then the set of 
matrices $\{M([I])\}_{I \in \Sigma^{(k)}}$ can be diagonalized simultaneously. That is, there exists a unique $P \in \mathbf{M}_{d \times d}(\mathbb{R})$ such that $P M([I]) P^{-1}:=D([I])$ is a diagonal matrix for all $I \in$ $\Sigma^{(k)}$. Since $H$ is primitive, there exist $L$ and $R>0$ such that (19) holds. We first compute the $L^{q}$-spectrum $\tau_{\mu}$, where $\mu \in \mathscr{M}_{T}\left(\Sigma_{A}\right)$ is defined in the proof of Theorem A with the property that there exists a constant $C^{\prime}>0$ such that for each $I \in \Sigma_{A, n}$,

$$
\frac{1}{C^{\prime}} \rho^{-n}\left\|\prod_{[I]} M\right\| \leq \mu([I]) \leq C^{\prime} \rho^{-n}\left\|\prod_{[I]} M\right\| .
$$

This induces

$$
\begin{aligned}
& \tau_{\mu}(q) \\
& =\frac{1}{\log \lambda} \lim _{n \rightarrow \infty} \frac{1}{n} \log \sum_{I \in \Sigma_{A, n}} \mu([I])^{q} \\
& =\frac{1}{\log \lambda}\left(-q \log \rho+\lim _{n \rightarrow \infty} \frac{1}{n}\right. \\
& \left.\times \log \sum_{I \in \Sigma_{A, n}}\left\|\prod_{l=0}^{n-1} M\left(\pi^{(k)}\left(T^{l}([I])\right)\right)\right\|^{q}\right) \\
& =\frac{1}{\log \lambda}\left(-q \log \rho+\lim _{n \rightarrow \infty} \frac{1}{n}\right. \\
& \left.\times \log \sum_{I \in \Sigma_{A, n}}\left\|P^{-1} \prod_{l=0}^{n-1} D\left(\pi^{(k)}\left(T^{l}([I])\right)\right) P\right\|^{q}\right) \\
& =\frac{1}{\log \lambda}\left(-q \log \rho+\lim _{n \rightarrow \infty} \frac{1}{n}\right. \\
& \left.\times \log \sum_{I \in \Sigma_{A, n}} \prod_{l=0}^{n-1} \rho\left(M\left(\pi^{(k)}\left(T^{l}([I])\right)\right)\right)^{q}\right) \\
& =\frac{1}{\log \lambda}(-q \log \rho+\log \Theta(q)) \text {. }
\end{aligned}
$$

We note here that the second equality comes from the positivity of $L, R$ and $P$ is invertible. Since $\tau_{\nu}(q)=\tau_{\mu}(q)=$ $\tau_{\phi_{*} \mu}(q)$, the proof is completed.

Here we give a concrete example for the dimension spectrum of sofic system.

Example 8. Let $\Sigma_{A}$ be the golden mean shift with

$$
A=\left[\begin{array}{ll}
1 & 1 \\
1 & 0
\end{array}\right]
$$

and the right-resolving sliding block code with $L=2$ :

$$
\Phi^{(2)}([00])=a, \quad \Phi^{(2)}([01])=b, \quad \Phi^{(2)}([10])=b .
$$

Define a matrix potential on $\mathbf{S}_{1}$, that is, $k=1$, as in Proposition 7 by

$$
N_{a}=\left[\begin{array}{ll}
1 & 1 \\
1 & 1
\end{array}\right], \quad N_{b}=\left[\begin{array}{ll}
1 & 0 \\
0 & 1
\end{array}\right] .
$$

Then $\Lambda=\{[00]\}$ and $M([00])=N_{a}>0$. A little modification of the proof of Theorem A indicates that $P_{N}(q)$ is differentiable. Suppose $\alpha=P_{N}^{\prime}(q)$ with $q \neq 0$; Theorem 6 is applied to show that

$$
\operatorname{dim}_{H} E_{N}(\alpha)=-\alpha q+P_{N}(q) .
$$

On the other hand, one can easily compute that

$$
\mathbf{R}(q)=\left[\begin{array}{cc}
2^{q} & 1^{q} \\
1^{q} & 0
\end{array}\right]
$$

and Proposition 7 applies to show that

$$
\begin{array}{r}
\tau_{v}(q)=\frac{1}{\log g}\left(-q \log (1+\sqrt{2})+\log \frac{2^{q}+\sqrt{2^{2 q}+4}}{2}\right), \\
\text { where } g=\frac{1+\sqrt{5}}{2} .
\end{array}
$$

4.2. Computation of Pressure. Let $\left(\Sigma_{A}, T\right)$ be a subshift of finite type and $P_{M}(q)$ be its pressure for $q \in \mathbb{R}$. If $P_{M}(q)$ is differentiable, Theorem 1.3 of [4] demonstrates that the dimension spectrum can be computed via the formula of $P_{M}(q)$. However, the computation of the explicit formula for $P_{M}(q)$ is not easy. If $(\mathbf{S}, S)$ a sofic system, we provide a wide class of matrix potential on $\mathbf{S}$ for which we can compute its $P_{N}(q)$ rigorously which leads to the dimension spectrum of $E_{N}(\alpha)$. We first give a theorem which is analogous to Theorem 1.3 of $[4]$.

Theorem 9. Let $N \in \Gamma_{+}^{H}(\mathbf{S})$. We have for any $\alpha=P_{N}^{\prime}(q)$ with $q \neq 0$

$$
\operatorname{dim}_{H} E_{N}(\alpha)=\frac{1}{\log \lambda}\left(-\alpha q+P_{N}(q)\right) .
$$

Proof. Up to a minor modification, the proof is identical to the proof of Theorem 1.3 of [4] and we omit it here.

We prove the following class for which we can compute its $P_{N}(q)$ and $\operatorname{dim}_{H} E_{N}(\alpha)$.

Theorem 10. If $N \in \Gamma^{C}(\mathbf{S})$ depends on $k$-coordinate, then it satisfies the following properties.

(1) Let $H=H^{(k)}$ be the matrix constructed in Theorem $A$ which is primitive.

(2) Let $M \in \Gamma^{C}\left(\Sigma_{A}^{(k)}\right)$ be induced from $N$ as in the proof of Theorem $A$. If there exists a sequence of real numbers $\chi=\left(\chi_{I}\right)_{I \in \Sigma_{A}^{(k)}}$ and $K \in \mathbf{M}_{1 \times d}(\mathbb{R})$ is a row vector such that for any $I \in \Sigma_{A}^{(k)}$ we have

$$
K M([I])=\chi_{I} K \text {. }
$$


Then

$$
P_{N}(q)=\log \Theta(q)
$$

where $\Theta(q)$ denotes the maximal eigenvalue of $\mathbf{F}(q) \in$ $\mathbf{M}_{q^{(k-1)} \times q^{(k-1)}}(\mathbb{R})$ defined in $(77)$ and thus it is differentiable. Furthermore, (74) can be computed explicitly.

Proof. Define $\mathbf{F}(q) \in \mathbf{M}_{q^{(k-1)} \times q^{(k-1)}}(\mathbb{R})$ by

$$
\mathbf{F}(q)_{I_{1}, I_{2}}= \begin{cases}\left(K M\left(\left[I_{1} \oplus I_{2}\right]\right) Y\right)^{q}, & \text { if } I_{1} \oplus I_{2} \in \Sigma^{(k)} \\ 0, & \text { otherwise }\end{cases}
$$

where $Y \in \mathbf{M}_{d \times 1}(\mathbb{R})$ is a column vector with $K Y=1$. Since $H$ is primitive, then the left and right eigenvectors are positive; that is, $L, R>0$. Combining (75) with Perron-Frobenius Theorem we have

$$
\begin{aligned}
& P_{N}(q) \\
& =\lim _{n \rightarrow \infty} \frac{1}{n} \\
& \times \log \sum_{J \in \mathbf{S}_{n}} \sup _{y \in[J]}\left\|N(y) N(S(y)) \cdots N\left(S^{n-1}(y)\right)\right\|^{q} \\
& =\lim _{n \rightarrow \infty} \frac{1}{n} \log \sum_{J \in \mathbf{S}_{n}}\left\|N(y) N(S(y)) \cdots N\left(S^{n-1}(y)\right)\right\|^{q} \\
& =\lim _{n \rightarrow \infty} \frac{1}{n} \\
& \times \log \sum_{I \in P_{J}, x \in I, \phi(x)=y}\left\|M(x) M(T(x)) \cdots M\left(T^{n-1}(x)\right)\right\|^{q} \\
& =\lim _{n \rightarrow \infty} \frac{1}{n} \log \sum_{I \in \Sigma_{A, n+L-1}}\left\|\prod_{l=0}^{n-1} M\left(\pi^{(k)}\left(\Phi_{k}\left(T^{l}([I])\right)\right)\right)\right\|^{q} \\
& =\lim _{n \rightarrow \infty} \frac{1}{n} \\
& \times \log \sum_{I \in \Sigma_{A, n+L-1}}\left(\chi_{\pi^{(k)}\left(\Phi_{k}([I])\right)} \cdots \chi_{\pi^{(k)}\left(\Phi_{k}\left(T^{n-1}([I])\right)\right)}\right)^{q} \\
& \text { (By (77)) } \\
& =\log \Theta(q) .
\end{aligned}
$$

The second equality exists because $N$ is finite-coordinate dependent and 4 th equality comes from the right-resolving property of $\phi$. This completes the proof.

Remark 11. (1) In Theorem A, we always assume that if one is regarded as $\Sigma_{A}=(G, E)$ where $G=\left\{I: I \in \Sigma^{(k-1)}\right\}$ and edges,

$$
E=\left\{\left(I_{1}, I_{2}\right): I_{1} \oplus I_{2} \in \Sigma^{(k)}\right\} .
$$

Then there is only one level from $I_{1}$ to $I_{2} \in \Sigma^{(k)}$; that is, the number of levels of $\left(I_{1}, I_{2}\right)$ for any $I_{1}$ and $I_{2}$ is equal to one, and thus $H^{(k)}$ can be constructed with the entry which is

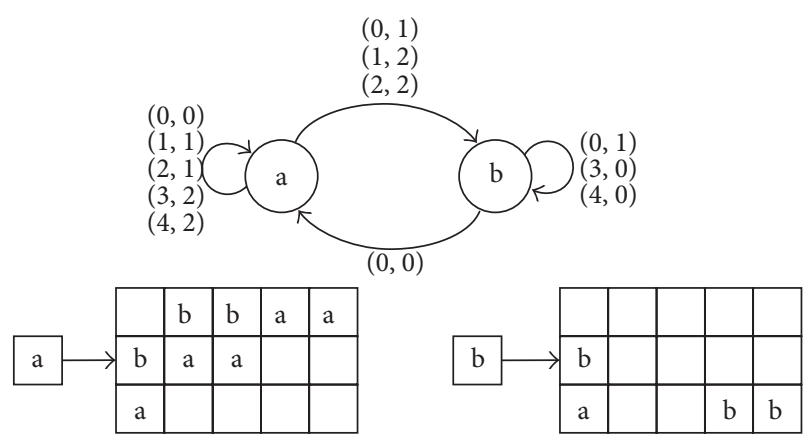

Figure 1: The substitution rules associated with $K_{T}$.

a single smaller matrix. However, if there is more than one level from $I_{1}$ to $I_{2}$, we only need to modify $H_{I_{1}, I_{2}}^{(k)}$ by

$$
H_{I_{1}, I_{2}}^{(k)}= \begin{cases}l\left(I_{1} \oplus I_{2}\right) M\left(\left[I_{1} \oplus I_{2}\right]\right), & \text { if } I_{1} \oplus I_{2} \in \Sigma^{(k)} \\ 0_{d \times d}, & \text { otherwise, }\end{cases}
$$

where $l\left(I_{1} \oplus I_{2}\right)$ denotes the number of levels from $I_{1}$ to $I_{2}$. Since $\phi$ is right resolving, Theorem A still follows.

(2) In the assumption (75) of Theorem 10, one can easily check that the result remains if there exists a column vector $K$ such that for any $I \in \Sigma_{A}^{(k)}$

$$
M([I]) K=\chi_{I} K \text {. }
$$

(3) One can also easily check that for those classes of Theorem 10, if $v \in \mathscr{M}_{S}(\mathbf{S})$ is the measure in Theorem A, Then the $L^{q}$-spectrum is

$$
\tau_{\nu}(q)=\frac{1}{\log \lambda}(-q \log \rho+\log \Theta(q)),
$$

where $\rho$ is the maximal eigenvalue of $H^{(k)}$ and $\Theta(q)$ is the maximal eigenvalue of (77).

In the following example, the computation of pressure helps for the computation of dimension spectrum of sofic affine-invariant set.

Example 12 (sofic affine-invariant set. See [15, Example 1]). Consider $\mathbb{T}^{2}=\mathbb{R}^{2} / \mathbb{Z}^{2}$ which is invariant under

$$
T=\left[\begin{array}{ll}
5 & 0 \\
0 & 3
\end{array}\right]
$$

Let $D=\{0, \ldots, 4\} \times\{0, \ldots, 2\}$ and for any $\left\{d_{k}\right\}_{k=1}^{\infty} \in D^{\mathbb{N}}$ the base $T$ representation is as follows (reader may refer to [7] for details):

$$
R_{T}\left(\left\{d_{k}\right\}\right)=\sum_{k=1}^{\infty}\left[\begin{array}{cc}
5^{-k} & 0 \\
0 & 3^{-k}
\end{array}\right] d_{k}
$$

Let $A$ be a matrix index by $D$ which is incidence matrix and $K_{T}(A)$ is also defined as the image of $R_{T}$; that is,

$$
K_{T}(A)=\left\{R_{T}\left\{d_{k}\right\}: A\left(d_{k}, d_{k+1}\right)=1 \text { for } k \geq 1\right\} .
$$




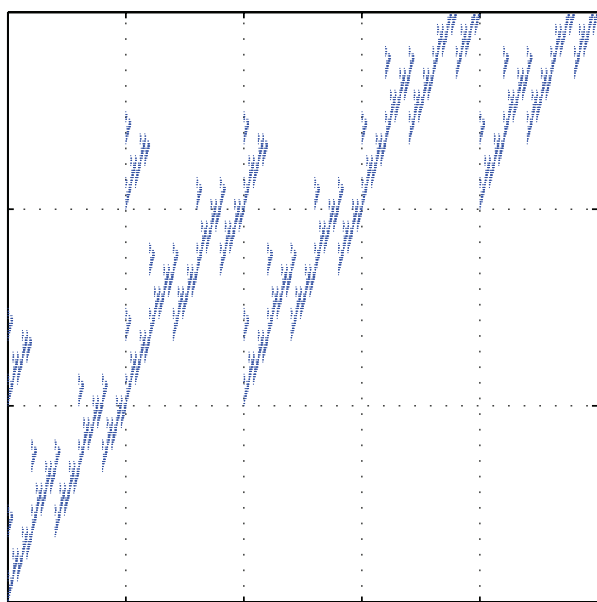

(a)

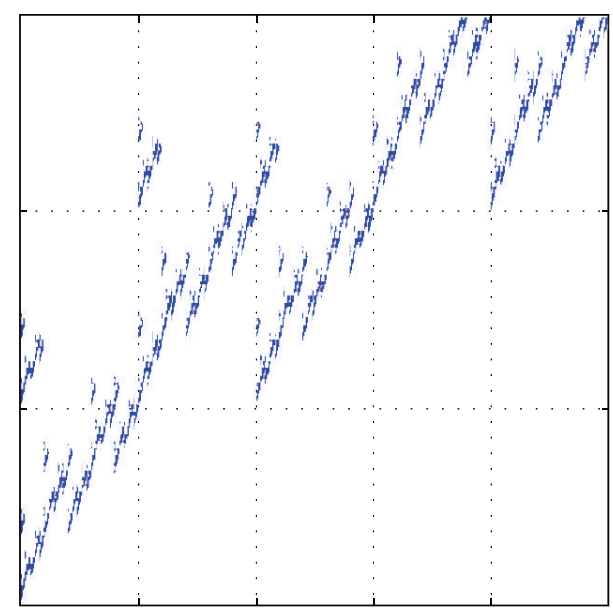

(b)

Figure 2: The first and second figures are fourth and seventh steps of iteration, respectively.

Let $\mathbf{S}$ be a sofic system that is induced by projecting $\Sigma_{A}$ on $y$-direction, see Figure 1, and let $N=(N([s]))_{s=0}^{2}$ be defined as

$$
\begin{gathered}
N([0])=\left[\begin{array}{ll}
1 & 0 \\
1 & 2
\end{array}\right], \quad N([1])=\left[\begin{array}{ll}
2 & 1 \\
0 & 1
\end{array}\right], \\
N([2])=\left[\begin{array}{ll}
2 & 2 \\
0 & 0
\end{array}\right] .
\end{gathered}
$$

Let

$$
\begin{gathered}
K_{T}^{(n)}(\mathbf{S})=\left\{R_{T}\left\{d_{k}\right\}:\left(d_{k}\right)_{k \geq 1} \in \mathbf{S}_{n}=\phi\left(\Sigma_{A, n+L-1}\right)\right\}, \\
K_{T}(\mathbf{S})=\left\{R_{T}\left\{d_{k}\right\}:\left(d_{k}\right)_{k \geq 1} \in \mathbf{S}=\phi\left(\Sigma_{A}\right)\right\} .
\end{gathered}
$$

$K_{T}^{(4)}(\mathbf{S})$ and $K_{T}^{(7)}(\mathbf{S})$ are represented in Figure 2. Define $\Phi^{(2)}$ by

$$
\begin{array}{cc}
\Phi^{(2)}([i 0])=[0] & \text { for } i=0,3,4, \\
\Phi^{(2)}([i 1])=[1] & \text { for } i=0,1,2, \\
\Phi^{(2)}([i 2])=[2] & \text { for } i=1,2,3,4 .
\end{array}
$$

Then one can easily check that if $K=(1,1)$, then $\chi=\left(\chi_{I}\right)_{I \in \Sigma_{2}}$ can be defined as

$$
\chi_{0}=\chi_{1}=\chi_{2}=2 .
$$

$H^{(1)}$ can be defined as follows ((1) of Remark 11):

$$
\begin{aligned}
& H^{(1)} \\
& \quad=\left[\begin{array}{cc}
N([0])+2 N([1])+2 N([2]) & N([1])+2 N([2]) \\
N([0]) & 2 N([0])+N([1])
\end{array}\right] .
\end{aligned}
$$

For any $q \in \mathbb{R}$, define $\mathbf{F}(q)$ from $H$ by taking $K=(1,1)$ and $Y=\left(\begin{array}{l}3 / 4 \\ 1 / 4\end{array}\right)$ :

$$
\mathbf{F}(q)=\left[\begin{array}{ll}
5 \times 2^{q} & 3 \times 2^{q} \\
1 \times 2^{q} & 3 \times 2^{q}
\end{array}\right]
$$

and then

$$
P_{N}(q)=\log \Theta(q)=\log 6+q \log 2 .
$$

Theorem 6 indicates that $\alpha=\log 2$ and

$$
\operatorname{dim}_{H} E_{N}(\log 2)=\frac{1}{\log 6}(-q \log 2+(\log 6+q \log 2))=1
$$

which is constant multifractal.

If $N$ is symmetric, we also have the following estimate.

Corollary 13. Let $N \in \Gamma^{C}(\mathbf{S})$ depend on $k$-coordinate and let $H=H^{(k)}$ be the matrix constructed in Theorem A which is primitive. Let $M \in \Gamma^{C}\left(\Sigma_{A}^{(k)}\right)$ be induced from $N$, if for any $I \in$ $\Sigma_{A}^{(k)}$, there exist $a_{I}, b_{I} \in \mathbb{R}$ such that

$$
M([I])=\left[\begin{array}{ll}
a_{I} & b_{I} \\
b_{I} & a_{I}
\end{array}\right] .
$$

Then

$$
P_{N}(q)=\log \Theta(q),
$$

where $\Theta(q)$ denotes the maximal eigenvalue of $\mathbf{F}(q) \in$ $\mathbf{M}_{q^{(k-1)} \times q^{(k-1)}}(\mathbb{R})$ which is defined by

$$
\mathbf{F}(q)_{I_{1}, I_{2}}= \begin{cases}\left(a_{I}+b_{I}\right)^{q} & \text { if } I:=I_{1} \oplus I_{2} \in \Sigma^{(k)} \\ 0 & \text { otherwise. }\end{cases}
$$

Proof. Since for any $I \in \Sigma_{A}^{(k)}$,

$$
\left[\begin{array}{ll}
1 & 1
\end{array}\right]\left[\begin{array}{ll}
a_{I} & b_{I} \\
b_{I} & a_{I}
\end{array}\right]=\left(a_{I}+b_{I}\right)\left[\begin{array}{ll}
1 & 1
\end{array}\right] .
$$

Theorem 10 is applied to show that $P_{N}(q)=\log \Theta(q)$, where $\Theta(q)$ is the maximal eigenvalue of $(96)$. The proof is completed. 
Example 14 (continued). Under the same substitution rule of Example 12, if the potentials on $\mathscr{A}(\mathbf{S})$ are as follows

$$
\begin{gathered}
N([0])=\left[\begin{array}{ll}
2 & 1 \\
1 & 2
\end{array}\right], \quad N([1])=\left[\begin{array}{ll}
1 & 3 \\
3 & 1
\end{array}\right], \\
N([2])=\left[\begin{array}{ll}
0 & 1 \\
1 & 0
\end{array}\right],
\end{gathered}
$$

one can easily check that $H^{(1)}$ is primitive and define

$$
\mathbf{F}(q)=\left[\begin{array}{cc}
3^{q}+2 \times 4^{q}+2 \times 1^{q} & 4^{q}+2 \times 1^{q} \\
3^{q} & 2 \times 3^{q}+4^{q}
\end{array}\right]
$$

Then

$$
P_{N}(q)=\log 2+\log \left(1+3^{q}+4^{q}\right)
$$

\section{Conflict of Interests}

The authors declare that there is no conflict of interests regarding the publication of this paper.

\section{Acknowledgment}

Ban is partially supported by the National Science Council, ROC (Contract no. NSC 102-2628-M-259-001-MY3). Chang is grateful for the partial support of the National Science Council, ROC (Contract no. NSC 102-2115-M-035-004-).

\section{References}

[1] W. Sierpiński, "Surune courbe cantorienne qui contient une image biunivoque et continue de toute courbe donne," Comptes Rendus, vol. 162, pp. 629-642, 1916.

[2] C. McMullen, "The Hausdorff dimension of general Sierpiński carpets," Nagoya Mathematical Journal, vol. 96, pp. 1-9, 1984.

[3] D. J. Feng, "Lyapunov exponents for products of matrices and multifractal analysis. II. General matrices," Israel Journal of Mathematics, vol. 170, pp. 355-394, 2009.

[4] D. J. Feng and K. S. Lau, "The pressure function for products of non-negative matrices," Mathematical Research Letters, vol. 9, no. 2-3, pp. 363-378, 2002.

[5] D. J. Feng, "Lyapunov exponents for products of matrices and multifractal analysis. I. Positive matrices," Israel Journal of Mathematics, vol. 138, pp. 353-376, 2003.

[6] B. Kitchens and S. Tuncel, "Finitary measures for subshifts of finite type and sofic systems," Memoirs of the American Mathematical Society, vol. 58, no. 338, pp. 1-68, 1985.

[7] R. W. Kenyon and Y. Peres, "Hausdorff dimensions of sofic affine-invariant sets," Israel Journal of Mathematics, vol. 94, pp. 157-178, 1996.

[8] V. Climenhaga and D. J. Thompson, "Intrinsic ergodicity beyond specification: $\beta$-shifts, S-gap shifts, and their factors," 2011, http://arxiv.org/abs/1011.2780.

[9] D. J. Thompson, "Irregular sets, the $\beta$-transformation and the almost specification property," Transactions of the American Mathematical Society, vol. 364, no. 10, pp. 5395-5414, 2012.

[10] P. Varandas, "Non-uniform specification and large deviations for weak Gibbs measures," Journal of Statistical Physics, vol. 146, no. 2, pp. 330-358, 2012.
[11] D. Lind and B. Marcus, An Introduction to Symbolic Dynamics and Coding, Cambridge University Press, Cambridge, UK, 1995.

[12] B. Kitchens, Symbolic Dynamics. One-Sided, Two-Sided and Countable State Markov Shifts, Springer, New York, NY, USA, 1998.

[13] D. J. Feng and W. Huang, "Lyapunov spectrum of asymptotically sub-additive potentials," Communications in Mathematical Physics, vol. 297, no. 1, pp. 1-43, 2010.

[14] J.-R. Chazottes and E. Ugalde, "Projection of Markov measures may be Gibbsian," Journal of Statistical Physics, vol. 111, no. 5-6, pp. 1245-1272, 2003.

[15] E. Olivier, "Uniqueness of the measure with full dimension on sofic affine-invariant subsets of the 2-torus," Ergodic Theory and Dynamical Systems, vol. 30, no. 5, pp. 1503-1528, 2010. 


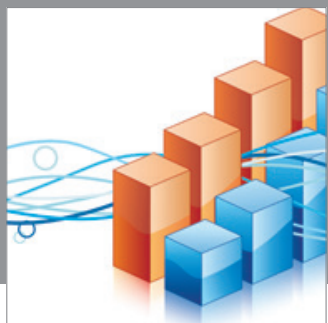

Advances in

Operations Research

mansans

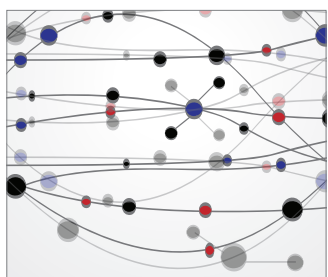

The Scientific World Journal
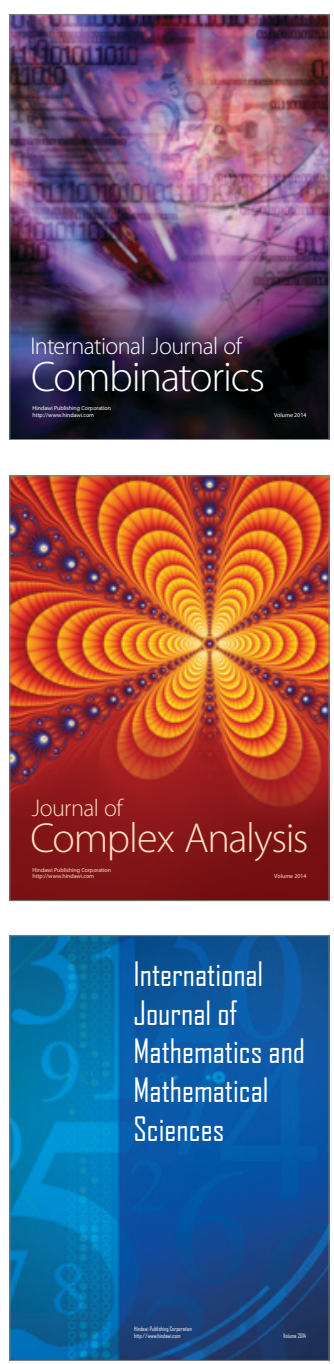
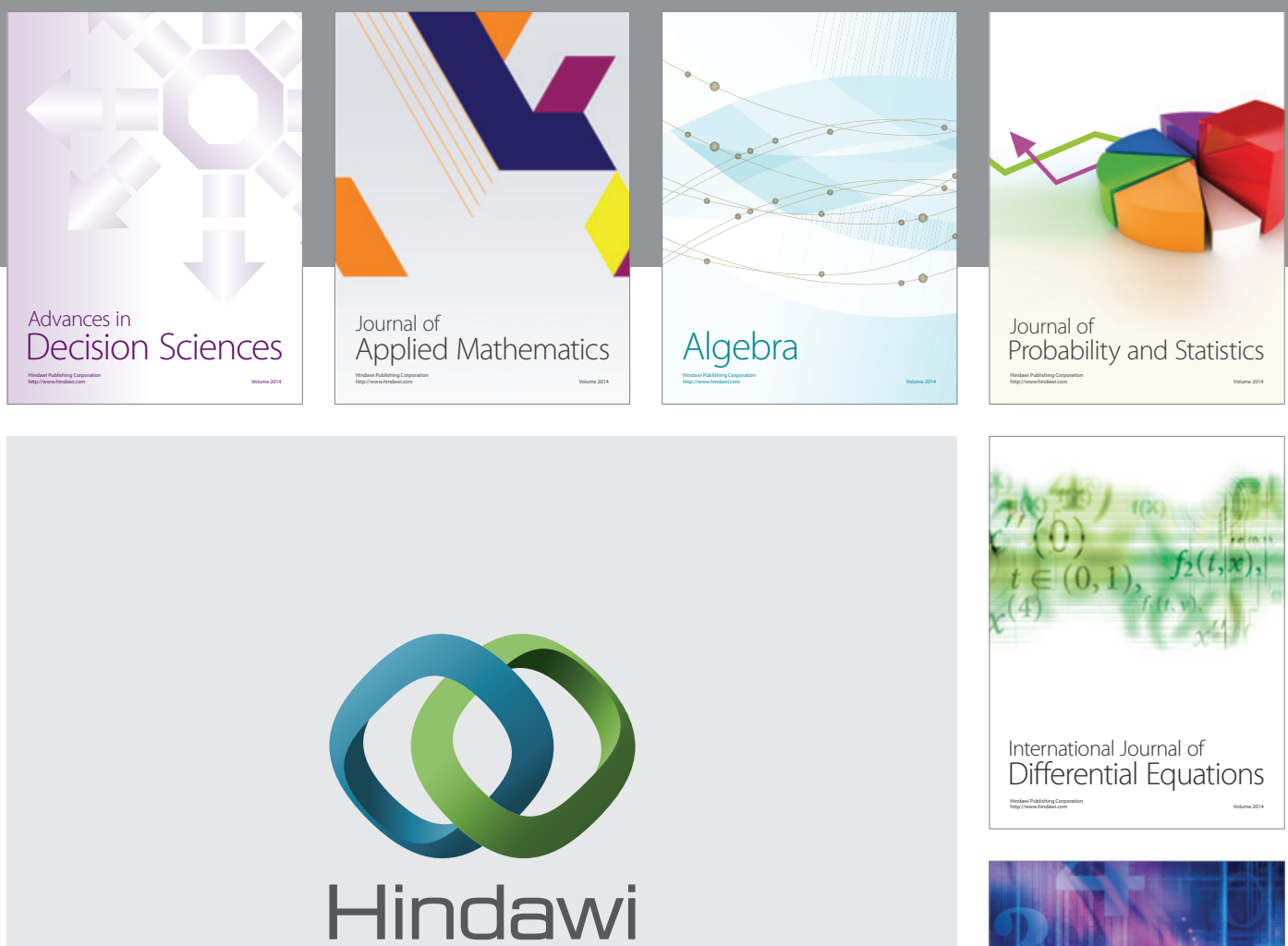

Submit your manuscripts at http://www.hindawi.com
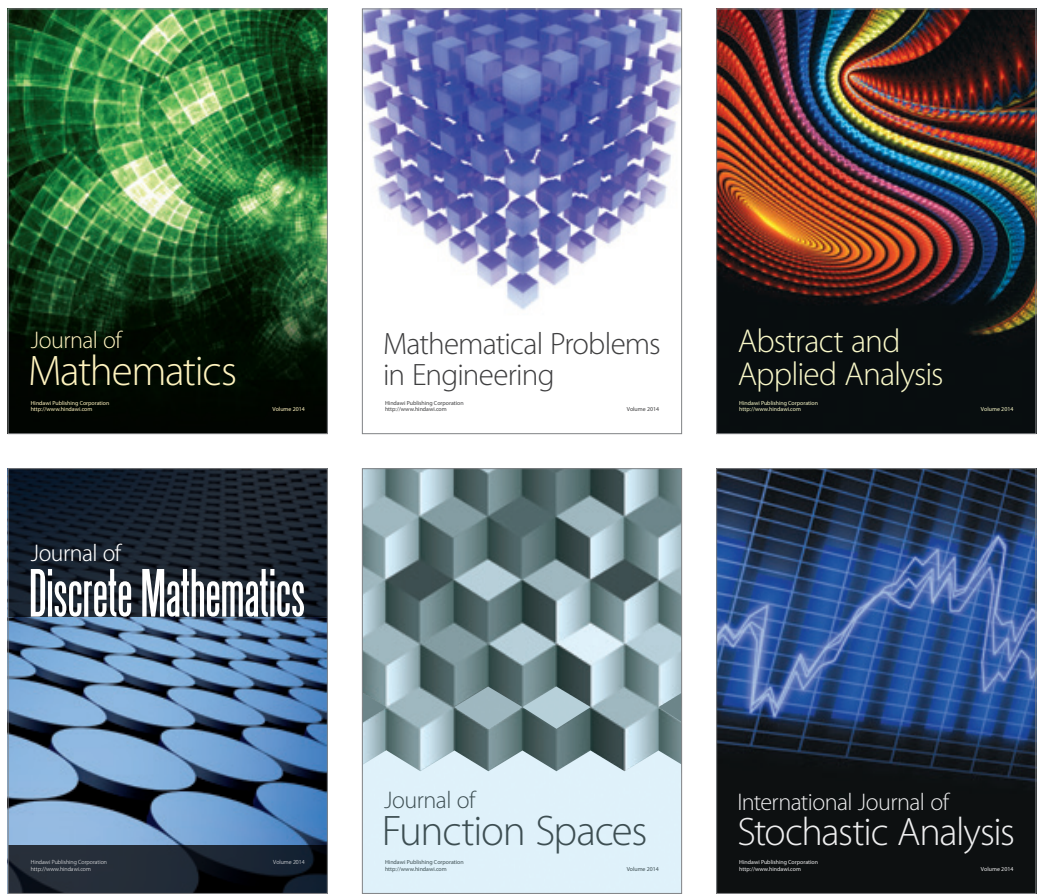

Journal of

Function Spaces

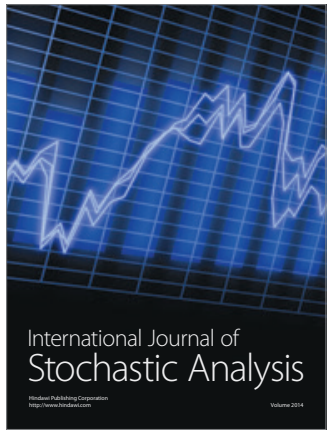

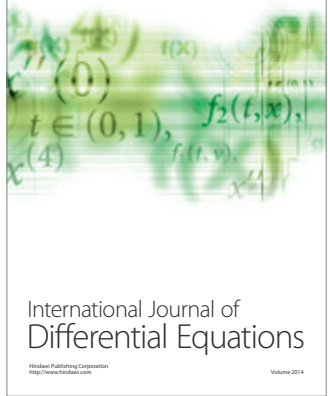
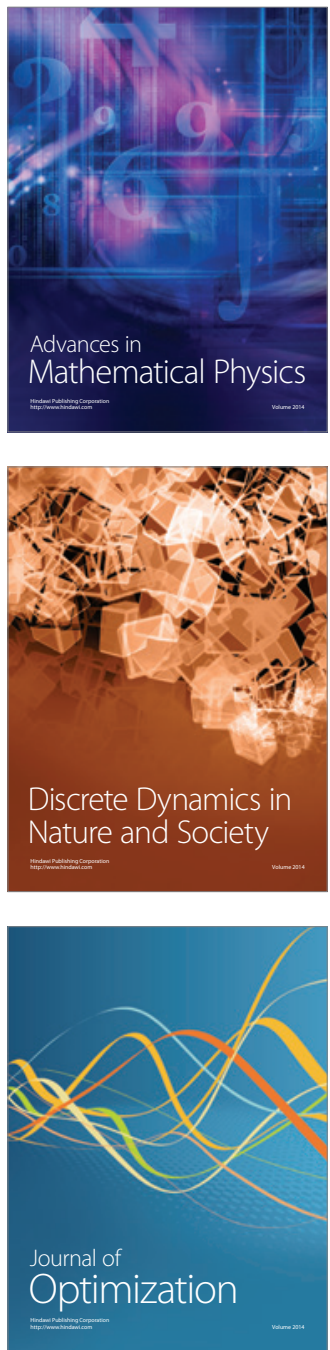